\title{
Measurement of induced surface charges, contact potentials, and surface states in GaN by electric force microscopy
}

\author{
P. M. Bridger, Z. Z. Bandić, E. C. Piquette, and T. C. McGill ${ }^{\text {a) }}$ \\ Thomas J. Watson, Sr. Laboratory of Applied Physics, California Institute of Technology, \\ Pasadena, California 91125
}

(Received 4 March 1999; accepted for publication 12 April 1999)

\begin{abstract}
We have studied molecular beam epitaxy grown $\mathrm{GaN}$ films of both polarities using electric force microscopy to detect sub $1 \mu \mathrm{m}$ regions of charge density variations associated with GaN extended defects. The large piezoelectric coefficients of $\mathrm{GaN}$ together with strain introduced by crystalline imperfections produce variations in piezoelectrically induced electric fields around these defects. The consequent spatial rearrangement of charges can be detected by electrostatic force microscopy and was found to be on the order of the characteristic Debye length for GaN at our dopant concentration. The electric force microscope signal was also found to be a linear function of the contact potential between the metal coating on the tip and GaN. Electrostatic analysis yielded a surface state density of $9.4 \pm 0.5 \times 10^{10} \mathrm{~cm}^{-2}$ at an energy of $30 \mathrm{mV}$ above the valence band indicating that the GaN surface is unpinned in this case. (c) 1999 American Institute of Physics.
\end{abstract} [S0003-6951(99)01223-1]

Nitride based devices have been of great interest in the last few years, notably due to their success in optoelectronics, where lasers and diodes have been demonstrated and successfully commercialized. ${ }^{1}$ Further applications of nitrides are expected in the arena of high power and high temperature devices, ${ }^{2-4}$ as well as solar blind ultraviolet detectors. ${ }^{5}$ It has been recently demonstrated that the large intrinsic piezoelectric coefficients of GaN and AlN are responsible for a high concentration two-dimensional electron gas at the $\mathrm{AlGaN} / \mathrm{GaN}$ interface in heterojunction field effect transistors (HFET). ${ }^{6,7}$ Other possibilities exist for the enhancement of electric properties of contacts to nitrides by piezoelectric engineering as recently demonstrated in the case of Schottky contacts. ${ }^{8}$ While most of the recent research has emphasized electronic device aspects of the piezoelectric effect, ${ }^{6-8}$ comparatively little work has concentrated on the investigation of fundamental properties and nanoscale characterization of piezoelectrically induced phenomena. One consequence of the piezoelectric effect is that it allows electrostatic force imaging of charge redistribution around defects due to local variations in strain caused by crystalline imperfections. Although the magnitude of the charge density is nonquantitative, electric force microscopy (EFM) can still provide interesting insight into the nature of defects, the piezoelectric effect in nitrides, as well as measurement of the surface state density and energy. ${ }^{9,10}$

The gallium nitride layers studied here were grown on c-plane sapphire substrates by radio frequency plasma assisted molecular beam epitaxy. Ga-polar GaN films were nucleated using AlN buffer layers whereas N-polar films were nucleated using a GaN buffer layer. Polarity was determined by reflection high-energy electron diffraction (RHEED) reconstruction at low temperature, ${ }^{11}$ and by $(\mathrm{KOH})$ etching. ${ }^{12,13}$ Other details of the growth conditions are presented elsewhere. ${ }^{14,15}$

${ }^{a)}$ Electronic mail: tcm@ssdp.caltech.edu
A variety of different metals were used for coating atomic force microscope (AFM) tips to vary the metal work function for electric force microscopy. Cobalt coated AFM tips were obtained commercially from Digital Instruments. Titanium, Al, Pt, Pd, Ce, W, and Au coated tips were fabricated in the following manner. Commercial silicon tapping mode AFM tips were plasma cleaned in a $30 \mathrm{~W}$ Ar plasma at a pressure of $8 \times 10^{-4}$ Torr. A $15 \mathrm{~nm}$ metal layer was then sputter deposited in sputter chamber at a base pressure of 1 $\times 10^{-7}$ Torr to provide the contact necessary for applying a bias to the tip.

The EFM data was collected using a Digital Instruments Nanoscope IIIa controller and a Bioscope scanning probe microscope operating in tapping mode. Electric force microscopy was performed in two ways: by detecting electrostatic forces and by detecting the surface potential. Surface potential measurements will be presented elsewhere. To detect the electrostatic forces, a voltage is applied to AFM tips coated with the above metals which are scanned across the surface at a constant tip-sample separation. Phase differences induced by electrostatic forces on the oscillating tip during scanning are detected and give a qualitative measurement of the local charge density.

When the metal tip is modeled as a small capacitive element, in similar fashion to Refs. 10,16, 17, the force it feels under an applied dc bias will be due to the chargecharge interaction, and changes in the capacitive energy,

$$
\text { Force }=\frac{q_{s} q_{t}}{4 \pi \epsilon_{0} z^{2}}+\frac{1}{2} \frac{d C}{d z}\left(V_{\text {applied }}-V_{\text {contact }}\right)^{2}
$$

where $d C / d z$ is the derivative of the sample-tip capacitance, $q_{s}$ is surface charge, $q_{t}$ is charge induced on the tip, and $V_{\text {contact }}=\phi_{m}-\chi_{\mathrm{GaN}}-\Delta E_{f n}-\Delta \phi$ is the contact potential between the tip metallization and $\mathrm{GaN}$ semiconductor. In this formula, $\phi_{m}$ is metal work function, $\chi_{\mathrm{GaN}}=4.2 \mathrm{eV}$ is the electron affinity of $\mathrm{GaN},{ }^{18} \Delta \phi$ is band bending caused by 

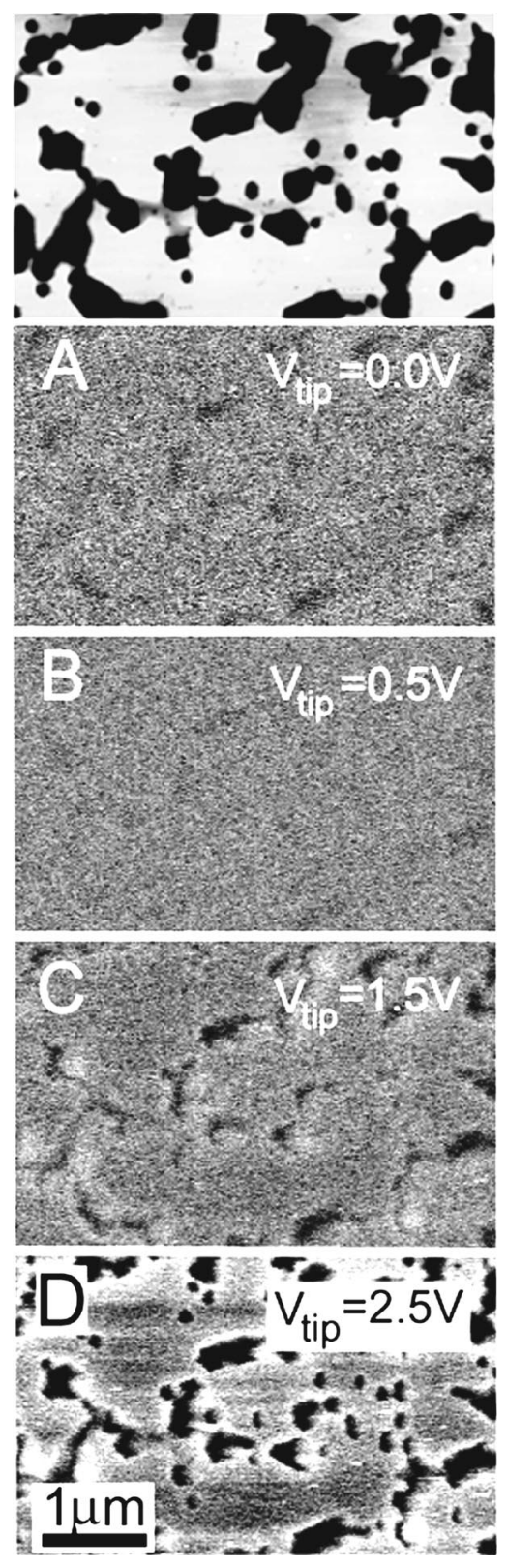

FIG. 1. Electrostatic force image of the surface of molecular beam epitaxy (MBE) grown $\mathrm{GaN}$ as a function of tip applied voltage for a Ga-polar sample. For reference, the top image is the AFM scan of the same area imaged with EFM. Scans A-D are the EFM data with the tip bias increasing from A-D. Notice the signal null at a tip bias of $0.5 \mathrm{~V}$. The tip sample separation was $50 \mathrm{~nm}$ in all the EFM images.

surface states, and $\Delta E_{f n}$ is the Fermi level position in the $\mathrm{GaN}$ referenced to the bottom of the conduction band.

For the experiments considered here, the tip-sample separation, $z$, was set at either 30 or $50 \mathrm{~nm}$.

The electrostatic forces were measured as a function of tip voltage to rule out topographical artifacts. Variation in the induced surface charges result in a force differential between the tip and the surface that increases with tip voltage which can be observed in the series of EFM images in Fig. 1. It was found that the electrostatic force was a function of the magnitude of the tip voltage and not the sign, consistent with the theoretical $V^{2}$ dependence. ${ }^{10,19}$ From Eq. (1), we can also observe a force minimum when the applied bias is equal to the contact potential between the GaN and the tip metallization. This was experimentally observed, and Fig. 1 illustrates Downloaded 03 Apr 2006 to 131.215.225.171. Redistribution subject to AIP license or copyright,

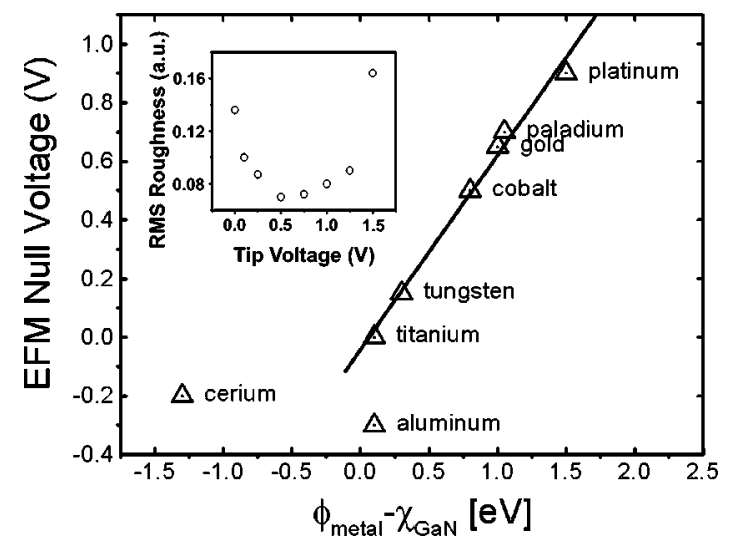

FIG. 2. Plot of the tip voltage for a minimum force condition vs the work function difference between the tip metallization and GaN. Inset: Plot of the rms contrast against the tip bias in arbitrary units illustrating the null condition for the cobalt coated tips.

this effect with a signal "null" at a tip voltage of $0.5 \mathrm{~V}$ in case of Co coated tips. The inset of Fig. 2 is a plot of the signal root-mean-square (rms) roughness (in arbitrary units) as a measure of contrast against the tip voltage to illustrate the minimum force condition. Typically, complete cancellation of the signal is not expected due to the first term in Eq. (1) leaving some residual image due to electrostatic forces.

Figure 2 is a plot of the measured tip null voltage $V_{\text {null }}$ as a function of the difference between metal work function and electron affinity of GaN. The observed dependence between $V_{\text {null }}$ and $\phi_{\text {metal }}-\chi_{\text {GaN }}$ is linear. Aluminum and cerium were found to be anomalous where the opposite sign of the voltage was required to null the EFM signal. This is attributed to different work functions of oxides which formed when the tips were exposed to air. A least squares linear fit of this experimentally observed dependence gives slope of 0.66 \pm 0.03 , and an intercept of $-0.04 \pm 0.03$. The slope is less than 1 and indicates the presence of surface states.

The situation will be considered as an electrostatic analysis of the following: (1) An ideal metal with with work function $\phi_{m}$. (2) A dielectric interface region with a thickness of the tip-sample separation. (3) A semiconductor surface with surface states up to a particular energy. An analysis of the barrier energy as a function of the metal work function and semiconductor electron affinity ${ }^{20}$ yields the following expression:

$$
\begin{aligned}
\phi_{B n} & =a\left(\phi_{m}-\chi\right)+(1-a)\left(\frac{E_{g}}{q}-\phi_{0}\right)-\Delta \phi \\
& =a\left(\phi_{m}-\chi\right)+b,
\end{aligned}
$$

where $\phi_{B n}$ is the barrier energy between a metal and an n-type semiconductor, $a$ and $b$ are slope and intercept, $\phi_{m}$ is the metal work function, $\chi$ is the electron affinity, $E_{g}$ is the band gap, $\Delta \phi$ is the barrier lowering, and $\phi_{0}$ is the energy of the surface states relative to the valence band. The density of surface states can now be expressed in terms of the regression coefficients, $a$ and $b$, as well as the dielectric constant, $\epsilon_{i}$, and thickness, $\delta$, of the metal-semiconductor interface:

$$
\phi_{0}=\frac{E_{g}}{q}-\frac{b+\Delta \phi}{1-a},
$$




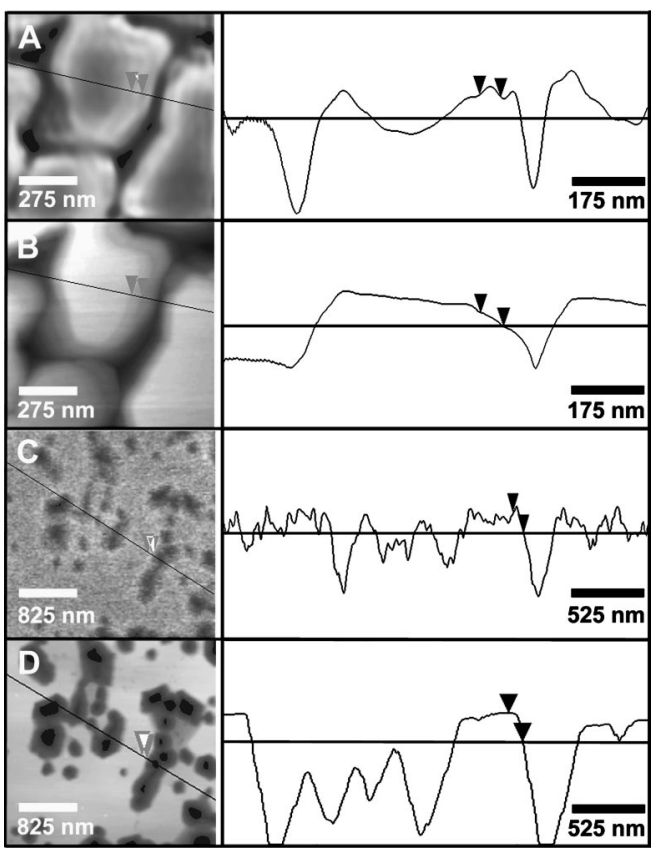

FIG. 3. (a) EFM image of the surface of $\mathrm{N}$-polar $\mathrm{GaN}$ and its associated line profile. The arrows indicate the $60 \mathrm{~nm}$ spatial extent of the screening charge associated with strain relaxation at the steps indicated in the following image. (b) The AFM and associated line profile for the same area in (a). Arrows indicate the steps of interest. (c) EFM of Ga-polar GaN and its associated line profile. Again, arrows indicate the spatial extent of the screening charge associated with the defect structure indicated by the AFM image in (d). In all cases the tip-sample separation was $30 \mathrm{~nm}$.

$$
D_{s}=\frac{(1-a) \epsilon_{i}}{a \delta q} .
$$

Substituting for the slope and intercept, the maximum surface state energy, $\phi_{0}$, lies $30 \mathrm{mV} \pm 90 \mathrm{mV}$ above the valence band. This is consistent with an observation of surface states at or below the valence band maximum using photoemission spectroscopy. ${ }^{21,22}$ Using an air gap as the dielectric for the interface, the calculated density of surface states is $9.4 \pm 0.5$ $\times 10^{10} \mathrm{~cm}^{-2}$. If water, $\epsilon_{i}=80 \epsilon_{0}$, is used as the dielectric to try to account for a typical surface contaminant, the surface state density becomes $7.5 \pm 0.4 \times 10^{12} \mathrm{~cm}^{-2}$. Since the density of chargeable defects required to pin the Fermi level is on the order of $10^{14} \mathrm{~cm}^{-2},{ }^{23}$ we conclude that the GaN surface is unpinned in this case.

Figure 3 shows that the nature of the charge rearrangement is due to screening of the piezoelectrically induced charges caused by strain relaxation at numerous defects. The N-polar [Figs. 3(a) and 3(b)] and Ga-polar [Figs. 3(c) and 3(d)] films have different defect structure and hence different surface morphology. Figures 3(a) and 3(b) show EFM and AFM image and the associated profile for N-polar film. We can observe steps (approximately $5 \mathrm{~nm}$ in height) on the Fig. 3(b), and associated charge accumulated on these steps [Fig. 3(a)]. In case of Ga-polar films, charge accumulation [Fig. $3(c)]$ is observed at the edges of the hexagonal pits. However, in both cases the strain relaxation and consequent charge rearrangement has a spatial extent of $60 \mathrm{~nm}$. A calculation of the Debye length gives $L_{D}=\sqrt{\epsilon_{s} k T / q^{2} N}$ $\leqslant 100 \mathrm{~nm}$ where both films have $N \geqslant 10^{15} \mathrm{~cm}^{-3}, \epsilon_{s}$ is the dielectric constant of $\mathrm{GaN}, q$ is the elementary charge, and $T=293 \mathrm{~K}$. The $60 \mathrm{~nm}$ spatial extent of the measured charge Downloaded 03 Apr 2006 to 131.215.225.171. Redistribution subject is within the experimental error in the determination of the doping density. Therefore, since the spatial extent of the charge density surrounding the defects is approximately equal to the Debye length within experimental error, it is believed that the observed charge is a screening charge rather than the bare surface charge density that would be induced on the surface due to the termination of the polarization of the film.

In summary, we have successfully demonstrated that EFM techniques can be used to detect local variations in piezoelectrically induced charge and potential on the sub 1 $\mu \mathrm{m}$ scale. These charges are believed to be screening charge on the surface since their spatial extent was comparable to the Debye length. We have also demonstrated that the EFM signal could be minimized by applying a voltage roughly equal to the contact potential between the tip metal and the $\mathrm{GaN}$. An analysis of this contact potential variation gave a surface state density of $9.4 \pm 0.5 \times 10^{10} \mathrm{~cm}^{-2}$ at an energy of $30 \mathrm{mV}$ above the valence band.

The authors would like to thank E. T. Yu and D. L. Smith for their useful comments and suggestions. This work was supported by DARPA/EPRI and monitored under Grant No. MDA972-98-1-0005.

${ }^{1}$ S. Nakamura, M. Senoh, S. Nagahama, N. Iwasa, T. Yamada, T. Matsushita, Y. Sugimoto, and H. Kiyoku, Appl. Phys. Lett. 70, 1417 (1997).

${ }^{2}$ M. A. Khan and M. S. Shur, Mater. Sci. Eng., B 46, 69 (1997).

${ }^{3}$ S. M. Mohammad and H. Morkoc, Prog. Quantum Electron. 20, 361 (1996).

${ }^{4}$ Z. Z. Bandić, E. C. Piquette, P. M. Bridger, R. A. Beach, T. F. Kuech, and T. C. McGill, Solid-State Electron. 42, 2289 (1998).

${ }^{5}$ J. M. Van Hove, R. Hickman, J. J. Klaassen, P. P. Chow, and P. P. Ruden, Appl. Phys. Lett. 70, 2282 (1997).

${ }^{6}$ G. Zandler, J. A. Majewski, M. Stadele, P. Vogl, and F. Compagnone, Phys. Status Solidi B 204, 133 (1997).

${ }^{7}$ A. Bykhovski, B. Gelmont, and M. Shur, J. Appl. Phys. 74, 6734 (1993).

${ }^{8}$ E. T. Yu, G. J. Sullivan, P. M. Asbeck, C. D. Wang, D. Qiao, and S. S. Lau, Appl. Phys. Lett. 71, 2794 (1998).

${ }^{9}$ Y. Martin, D. W. Abraham, and H. K. Wickramasinghe, Appl. Phys. Lett. 52, 1103 (1988).

${ }^{10}$ Dror Sarid, Scanning Force Microscopy (Oxford University Press, New York, 1991).

${ }^{11}$ A. R. Smith, R. M. Feenstra, D. W. Greve, M.-S. Shin, M. Skowronski, J. Neugebauer, and J. E. Northrup, Appl. Phys. Lett. 72, 2114 (1998).

${ }^{12}$ M. Seelmann-Eggebert, J. L. Weyher, H. Obloh, H. Zimmermann, A. Rar, and S. Porowski, Appl. Phys. Lett. 71, 2635 (1997).

${ }^{13}$ J. M. Van Hove, M. F. Rosamond, R. Hickman II, J. J. Klaassen, C. Polley, A. Wowchak, and P. P. Chow, presented at the 17th North American Conference on MBE, Penn State, 1998, talk G.3., J. Vac. Sci. Technol. $\mathrm{B}$ (to be published).

${ }^{14}$ E. C. Piquette, P. M. Bridger, Z. Z. Bandić, and T. C. McGill, presented at the 17th North American Conference on MBE, Penn State, 1998, talk G.1, J. Vac. Sci. Technol. B (to be published).

${ }^{15}$ E. C. Piquette, P. M. Bridger, Z. Z. Bandić, and T. C. McGill, Mater. Res. Soc. Symp. Proc. 512, 387 (1998).

${ }^{16}$ W. Nabhan, B. Equer, A. Broniatowski, and G. DeRosny, Rev. Sci. Instrum. 68, 3108 (1997).

${ }^{17}$ M. Nonnenmacher, M. P. O'Boyle, and H. K. Wickramasinghe, Appl. Phys. Lett. 58, 2921 (1991).

${ }^{18}$ Q. Z. Liu, and S. S. Lau, Solid-State Electron. 42, 677 (1998).

${ }^{19}$ F. Saurenbach and B. D. Terris, Appl. Phys. Lett. 56, 1703 (1990).

${ }^{20}$ S. M. Sze, Physics of Semiconductor Devices (Wiley, New York, 1981), pp. 270-275.

${ }^{21}$ K. E. Smith, S. S. Dhesi, C. B. Stagarescu, J. Downes, D. Doppalapudi, and T. D. Moustakas, Mater. Res. Soc. Symp. Proc. 482, 787 (1998).

${ }^{22}$ K. E. Smith, S. S. Dhesi, L. C. Duda, C. B. Stagarescu, J. H. Guo, J. Nordgren, R. Singh, and T. D. Moustakas, Mater. Res. Soc. Symp. Proc. 449, 787 (1998).

${ }^{23}$ A. Zur, T. C. McGill, and D. L. Smith, Phys. Rev. B 28, 2060 (1983). to AIP license or copyright, see http://apl.aip.org/apl/copyright.jsp 